\title{
Load Forecasting in Electrical Distribution Grid of Medium Voltage
}

\author{
Svetlana Chemetova ${ }^{1}$, Paulo Santos ${ }^{1}$ and Mário Ventim-Neves ${ }^{2}$ \\ ${ }^{1}$ Dep. of Electrical Engineering ESTSetúbal, Polytechnic Institute of Setúbal. \\ Rua Vale de Chaves Estefanilha, 2910-761 Setúbal Portugal. \\ ${ }^{2}$ Dep. of Electrical Engineering, Universidade Nova de Lisboa, Faculty of Sciences and \\ Technology Quinta da Torre, 2829-516 Caparica Portugal. \\ svetlana.chemetova@estsetubal.ips.pt,paulo.santos@estsetubal.ips.pt, ventim@uninova.pt
}

\begin{abstract}
The importance of forecasting has become more evident with the appearance of the open electricity market and the restructuring of the national energy sector.

This paper presents a new approach to load forecasting in the medium voltage distribution network in Portugal. The forecast horizon is short term, from 24 hours up to a week. The forecast method is based on the combined use of a regression model and artificial neural networks (ANN).

The study was done with the time series of telemetry data of the DSO (EDP Distribution) and climatic records from IPMA (Portuguese Institute of Sea and Atmosphere), applied for the urban area of Évora - one of the first Smart Cities in Portugal.

The performance of the proposed methodology is illustrated by graphical results and evaluated with statistical indicators. The error (MAPE) was lower than 5\%, meaning that chosen methodology clearly validate the feasibility of the test.
\end{abstract}

Keywords: Electric power systems, load forecasting, smart-grids, distribution systems, electric substations, artificial neural networks.

\section{Introduction}

The recognition of future electricity consumption patterns is an important part of the planning, operation and exploration of Electrical Power System. The quality control of Power Systems and effectiveness of its operation are very sensitive to forecasting errors. Thus, estimating the future energy consumption correctly is a mandatory prerogative in production management, transport and distribution of that energy.

The importance of load forecasting has grown with the emergence of the open electricity market, in the $90 \mathrm{~s}$ of the last century. The vertical structure (which was traditional) of the electricity sector was restructured. Relations between production companies, transportation, distribution and marketing in this new scenario became more complex both from a technical and from a commercial point of view.

In order to improve the efficiency and reliability of the distribution sector, Distribution Management System (DMS) applications are developed, that monitor and control the electricity distribution system [1]. Those applications are illustrated in Figure 1, in the form of a diagram. 


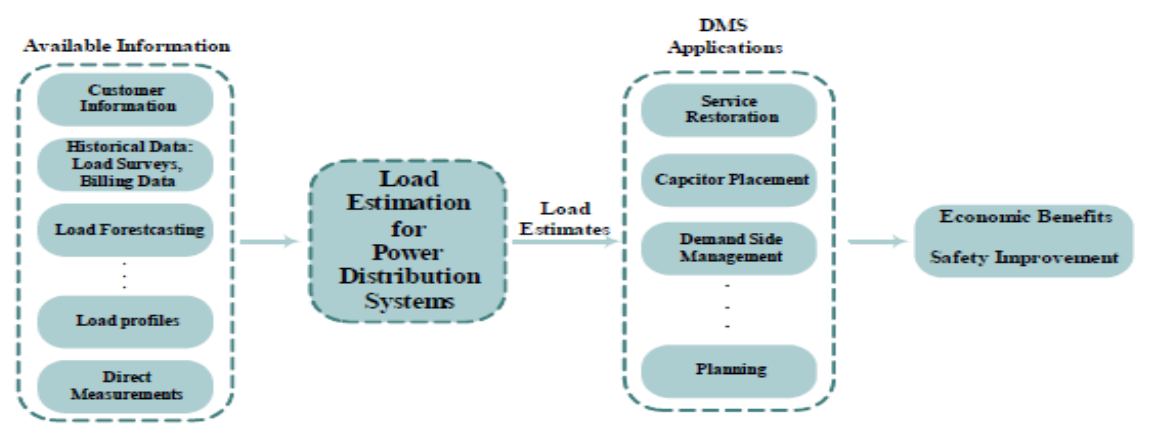

Fig. 1. The main functions of the Distribution Management Systems (taken from [1]).

Load Forecasting is considered in this context as one of the most important functions for the Power System operators. Among the applications, the most expected development is the Demand Side Management (DSM).

As an example of electric demand figure 2 represents two load curves, real and forecasted, made by the System Operator (SO) on December 13, 2012 [3]. This load refers to the Portuguese national transmission grid operator REN, without consideration of the interconnection with the European grid.

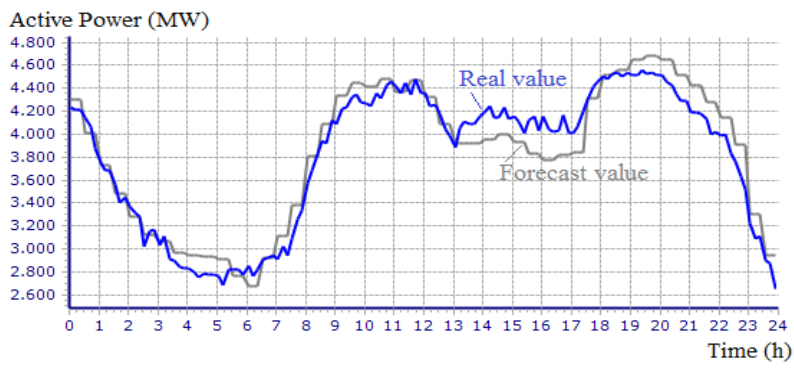

Fig. 2. Real and Forecast values collected in to the REN.

These load curves were obtained for all REN busbars including all the tree levels of voltage $(400 \mathrm{kV}, 220 \mathrm{kV}$ and $150 \mathrm{kV})$. The data were acquired in the most important substations using data acquisition system SCADA.

Electrical power is delivered to the Distribution Network, operated by EDP Distribution, which next performs the $60 / 30 \mathrm{kV}, 60 / 15 \mathrm{kV}$ and $60 / 10 \mathrm{kV} \mathrm{HV} / \mathrm{MV}$ transformation in the substations located in the main consumption centers.

Short-term load forecast allows the System Operator to answer to issues such as: network reconfiguration, voltage control, scheduling of maintenance actions and power factor correction, amongst others.

In Portugal there are few studies of load forecasting in distribution networks in medium voltage [5],[6]. Our work will try to contribute to fill this gap. This paper deals with a methodological approach, based on ANN, to forecast the next-hour load, applied to the distribution grid of medium voltage $(15 \mathrm{kV})$, located in the urban area of the city of Évora. 


\section{Relationship to cyber-physical systems}

To test and evaluate the developed load forecasting model, the choice fell on the urban area of the city of Évora, in the context of development of smart grids in this city, a pilot project carried out by EDP Distribution, called InovGrid [2].

The energy sector is facing several challenges: capacity to meet demand for electricity, security of supply, energy efficiency and reduced environmental impact. Évora has been giving priority to smart, sustainable and inclusive projects that contribute to achieving the objectives of Strategy Europe 2020 [4].

The European Smart Grid Task Force defines smart grids as energy networks that can efficiently integrate the behaviour of all users connected to them in order to ensure an economically efficient, sustainable power system with low losses and high quality and security of supply and safety:

The InovGrid project aims to move towards a system of intelligent electrical distribution, focused on energy telemanagement, which revolutionized the networks and their style of consumers / producers interaction.

This proposed change was implemented by a technological renovation and organizational adequacy of the distribution system operation and relationship with other stakeholders, based on a infrastructure that aims to respond to the needs arising from energy efficiency, remote management, distributed generation and microgeneration, and assume active control of the intelligent network (figure 3).

This change is associated with the installation of measuring equipment for metering. By the end of 2010, about 30 thousand low voltage customers (domestic, small trade and industry), covering the entire city of Évora, were linked to this integrated, intelligent electrical system.

The time series of the electrical energy consumption obtained by InovGrid telemetry system will be an essential database for the development of load forecasting applications. Load forecasting models, object of this study, will use these data.

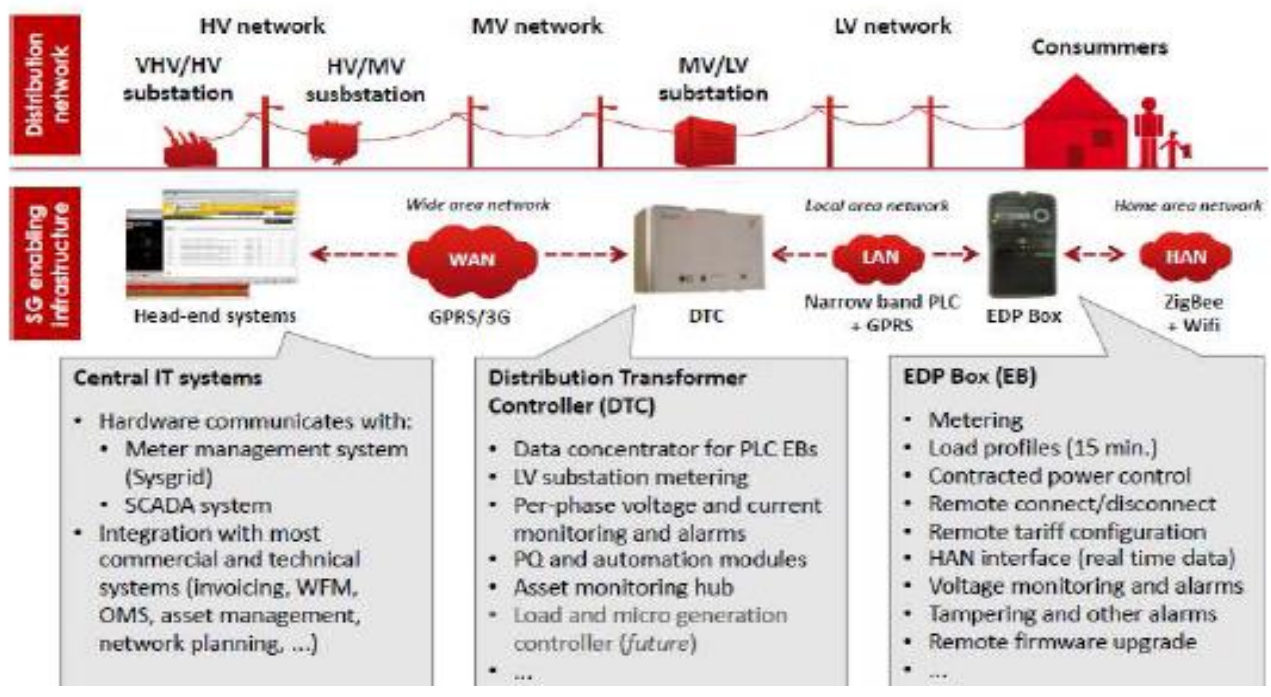

Fig. 3. Supervision and control of the distribution network, InovGrid Project (taken from [7]). 


\section{Data Analysis}

The urban area of the city of Évora is fed with $15 \mathrm{kV}$ medium voltage, by two primary substations (Évora and Caeira) connected to REN - ÉVORA transmission substation (Fig 4.).

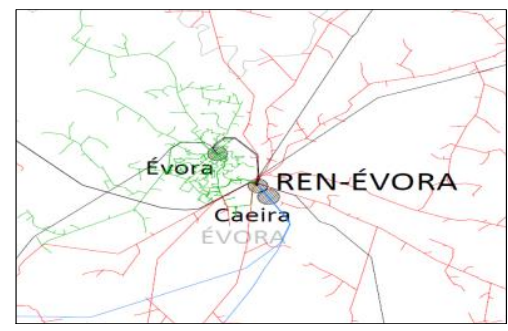

Fig. 4. HV and MV distribution networks around Évora.

In the substations, HV/MV transformers are installed: two $60 / 15 \mathrm{kV}$ in Évora substation and two 60/30/15 kV in Caeira substation (Fig.5.).

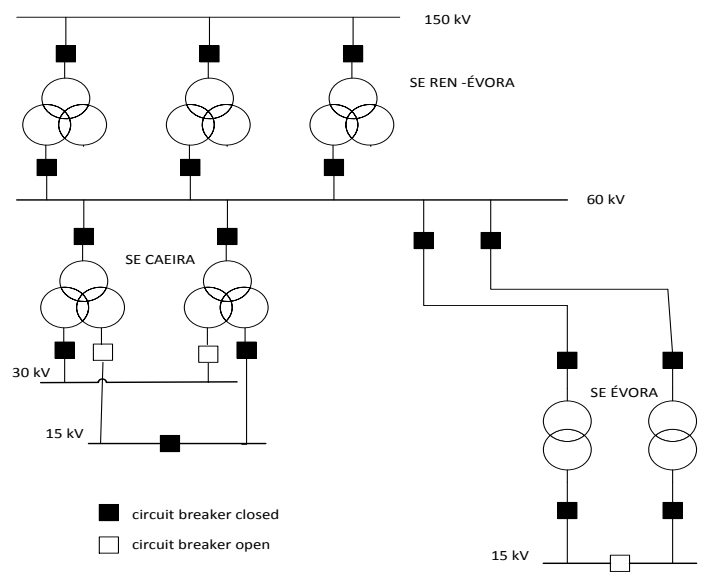

Fig. 5. One line diagram of HV substations in city of Évora.

The Évora substation has two transformers of 31.5 MVA each. This substation is located in an urban area. The substation of Caeira also has two power transformers of 31.5 MVA each and is situated on the border of the city, in the industrial zone. Among the Évora City consuming sectors prevail domestic, services and industry.

The data that are part of the "historic" set of endogenous variables (load) were collected at $15 \mathrm{kV}$ windings of each transformer, through the energy metering system during the years 2002-2013.

Among the social factors that affect the load diagram are the holidays, and other atypical days such as the football world championships etc.

The climatic factors that influence energy consumption are temperature, relative humidity, wind speed, rainfall and luminosity (collected from IPMA). 


\section{Forecast Methodology}

Forecasting methodologies have registered an evolution, over the past four to five decades. They started with the regressive approaches based on time series of active power [8]. The evolution of the electric market increased the complexity of the electrical energy systems and therefore the models based on regressions approaches became more and more complex. Methodologies based on analysis of uncertainty deal better with the information absence, reflecting sometimes more clearly the registered changes in the electrical power systems [9].

In resume, the development of methodologies for forecasting experienced a significant advance. In the sixties years of the XX century, methodologies mainly based on the regressive approach emerged; in the eighties and early nineties of the last century, methodologies emerged, that are based on knowledge and fuzzy techniques [10],[11], artificial neural networks [12],[13], hybrid systems [14] and genetic algorithms [15].

The methods for load prediction used in the energy industry in last years are mainly based on Artificial Intelligence theories because they allow the best way to deal with uncertainty, as well as non-linear functions. There are several scientific publications that prove the quality and robustness of the predictions based on these theories, specifically in artificial neural networks (ANN) [13],[16],[17].

In this research a standard feedforward backpropagation (BP) ANN is used. The neural network is trained on input data as well as the associated target values. The trained neural network can then make predictions based on the relationships learning during training. The data employed for training and validation the neural network were obtained from EDP Distribution (see Part 3 of this paper) for the year 2013. For the simulation data is used the year of 2014.

To define the structure and composition of the input vector to the model based on ANN it is necessary to follow a sequence of tasks [16]. Initially, data pre-processing is performed, mainly in order to fill the gaps caused by failures occurred in the data acquisition system operation. Next, a correlation analysis is made, between the endogenous and exogenous variables, to identify relevant influences of independent random factors on consumption dynamics. Endogenous variables are active and reactive power, exogenous variables are meteorological (among exogenous factors temperature is the most significant). Then, active power time series is subjected to an entropy analysis in order to determine the length of the series of short-term memory. This is to identify the maximum length of the relevant data sequence to be included in the input vector in the ANN algorithm. In a last phase, an auto-correlation analysis is carried out backwards in the time series of power, looking for the best correlation coefficients in such a way as to identify short sequences of data representing the presumable trend of consumption evolution. This is based on the behavior of load in homologous periods of past weeks [17].

After making the analysis described above, we concluded that practically there is no correlation between the load and the climatic variables, and we defined the following input vector, consisting of 13 endogenous variables: $[\mathrm{P}(\mathrm{t}-1), \mathrm{P}(\mathrm{t}-2), \mathrm{P}(\mathrm{t}-3), \mathrm{P}(\mathrm{t}-23), \mathrm{P}(\mathrm{t}-$ 24), $\mathrm{P}(\mathrm{t}-25), \mathrm{P}(\mathrm{t}-167), \mathrm{P}(\mathrm{t}-168), \mathrm{P}(\mathrm{t}-169), \Delta_{1}, \Delta_{2}, \Delta_{3}$ e $\left.\Delta_{4}\right]$. Figure 6 shows the predicted variable of the load and two contiguous variables in example of daily load diagram of Évora. The variables $\mathrm{P}(\mathrm{t}-1), \mathrm{P}(\mathrm{t}-2) \ldots \mathrm{P}(\mathrm{t}-\mathrm{n})$ are load values used in the construction of 
the input vector. $\Delta_{\mathrm{i}}$ gradient is calculated for the adjacent load values $\mathrm{P}(\mathrm{t}-\mathrm{k}), \mathrm{P}(\mathrm{t}-\mathrm{k}+1)$ where $\mathrm{k}$ is the "backward displacement".

For building the forecast model it was used a standard backpropagation feedforward ANN having a fully connected architecture with a single hidden layer. The number of neurons in the single hidden layer was half of the one of the input layer [17]. The hyperbolic tangent function was chosen for the middle layer. A linear function was used for the output layer. The input vector was normalized between -1 and 1 . This is a well-proven arrangement, adequate when, as in the present case, the relations between the variables at stake have a strong non-linear behavior. In many short-term forecast models this type of ANN structure is widely used [18].

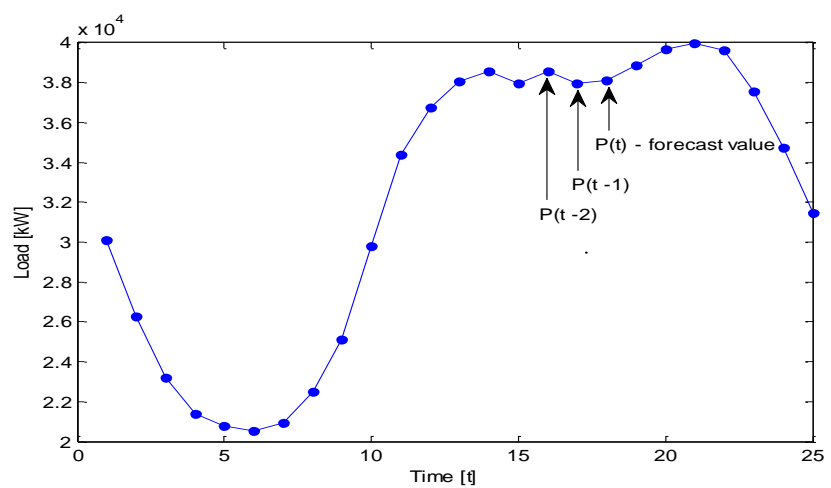

Fig.6. Representation of contiguous endogenous variables.

\section{Results}

The results obtained from simulation with the trained neural network are presented below in graphical form (Fig.7,8). Each graph shows a plot of both the "real" (obtained from telemetric reading) and "forecast" data.

The performance of the forecast model has to be evaluated; for this purpose the most current statistical indicators were obtained:

$$
\begin{array}{ll}
\text { Forecast error: } & \mathrm{e}_{\mathrm{t}}=\mathrm{P}_{\mathrm{t}}-\widehat{\mathrm{P}}_{\mathrm{t}} \\
\text { Mean error: } & \mathrm{ME}=\sum_{\mathrm{t}=1}^{\mathrm{n}} \frac{\mathrm{e}_{\mathrm{t}}}{\mathrm{n}} \\
\text { Percentage error: } & \mathrm{PE}_{\mathrm{t}}=\frac{\left(\mathrm{P}_{\mathrm{t}}-\widehat{\mathrm{P}}_{\mathrm{t}}\right)}{\widehat{\mathrm{P}}_{\mathrm{t}}} 100 \\
\text { Mean absolute percentage error: } & \mathrm{MAPE}=\sum_{\mathrm{t}=1}^{\mathrm{n}} \frac{\left|\mathrm{PE}_{\mathrm{t}}\right|}{\mathrm{n}}
\end{array}
$$

The forecast error $e_{t}$ is the difference between the load value recorded in the data acquisition process and the numerical result of our forecasting model. The maximum percentage error $\left(\mathrm{PE}_{\mathrm{t}}\right)$ calculated value is $6.26 \%$, the same for the first day and the first week, and is $7.64 \%$ for the first two months of the year 2014 (at this moment no more data referring to consecutive 2014 days is available). The mean absolute percentage error (MAPE) is the most significant indicator in these studies. MAPE calculated value 
is $1.7 \%, 1.3 \%$ and $1.07 \%$ for the same periods of time for 2014 , respectively. These are satisfactory results, considering that the maximum MAPE value in such models should not exceed $5 \%$.

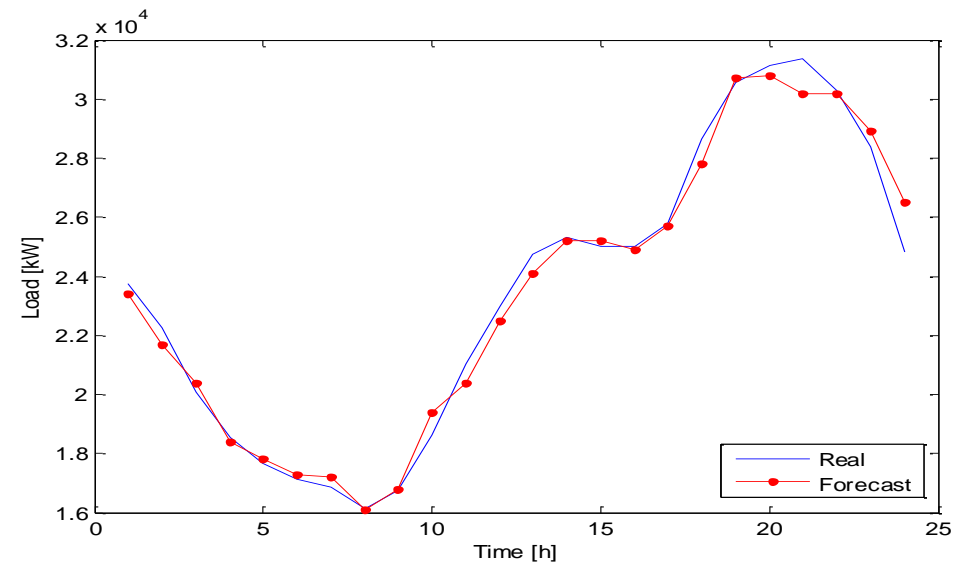

Fig.7. Load diagram of January $1^{\text {st }}, 2014$.

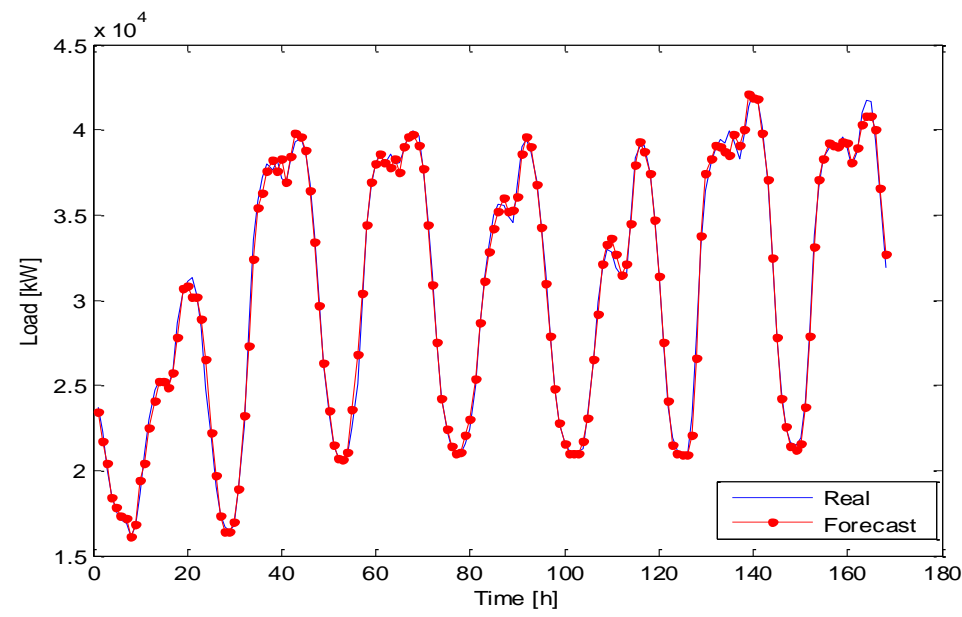

Fig. 8. Load diagram of first week of January, 2014.

\section{Conclusions and Future Work}

Short-term load forecasting assumes great importance in distribution networks, especially considering the smart grid environment. In fact, the distributors or retailers activities closely related to network management or energy purchase decisions might largely benefit from load forecast models in short time range. The time series of the electrical energy consumption obtained by InovGrid telemetry system used in conjunction with the values acquired by the SCADA system will constitute an essential database for implementation of load forecasting models. 
The innovative nature of the forecasting methodology in this work consists in the search for the best technique to achieve a better prediction, for the electrical medium voltage distribution grid.

Forecasting model based on ANN suggested in this work shows good results with very satisfactory error values.

As a future step we will develop a methodology to predict the maximum power daily load diagram (load peak forecasting), which will serve as a support application for operators of Electrical Power Systems.

Acknowledgments. The authors would like to thank EDP Distribuição and IPMA.

\section{References}

1. Wan J.: Nodal load estimation for electric power distribution systems. Drexel (2003)

2. Smart Energy Grid, http://www.inovgrid.pt/en accessed in November 2015

3. National Energetic Networks REN, 2012, http://www.ren.pt/ accessed in December 2012

4. GRID INNOVATION online, http://www.gridinnovation-on-line.eu/Articles/Library/InovGrid-Project---EDP-DistribuicaoPortugal.kl accessed in November 2015

5. Fidalgo, J.N.: Load curve estimation for distribution systems using ANN, pp. 197-202 Proc. of the $7^{\text {th }}$ WSEAS - CIMMACD'08, (2008)

6. Guimarães, A.: Forecast of medium term load evolution in distribution networks, IST (2008)

7. Loureiro M.: Smart grids: an integrated perspective on efficiency, from supply to demand. Coimbra (2014)

8. Heinemann, G. T., Nordman, D.A., Plant, E.C.: The Relationship Between Summer Weather and Summer Loads - A Regression Analysis. IEEE Transactions on Power Apparatus and Systems (1966)

9. Gross, G., Galiana, F.: Short term load forecasting, pp. 1558--1573. IEEE Proceedings, 75 (12), (1987)

10. Rahman, S.: Formulation and analysis of a rule-based short-term load forecasting algorithm. pp. 805--816. IEEE Proceedings, 78 (5), (1990)

11. Hsu, Y., Ho, K.: Fuzzy expert systems: an application to short term load forecasting, pp. 471--477. IEE Proceedings-C, 139 (6), (1992)

12. Park, D., El-Sharkawi, M., Marks, I. R.: Electric load forecasting using an artificial neural network, pp. 442--449. IEEE Transactions on Power Systems, 6 (2), (1991)

13. Marin, F., Garcia-Lagos, F., Joya, G., Sandoval, F.: Global model for short-term load forecasting using artificial neural networks, pp. 121--125. IEEE Proceedings on Generation, Transmission and Distribution, (2), (2002)

14. Sfetsos, A.: Short-term load forecasting with a hybrid clustering algorithm, pp. 257--262. IEEE Proceedings on Generation, Transmission and Distribution, 150 (3), (2003)

15. Maifeld, T., Sheblé, G: Short-term load forecasting by a neural network and refined genetic algorithm, pp. 147--152. Electric Power Systems Research, 31, (1994)

16. Hippert, H., Pereira, C., Souza R.: Neural networks for short-term load forecasting: A review and evaluation, pp. 44--55. IEEE Trans. on Power Systems. 16 (1), (2001)

17. Santos, P., Martins, A., Pires, A.: Designing the input vector to ANN-based models for shortterm load forecast in electricity distribution systems, pp.338-347. ELSEVIER Electrical Power and Energy Systems, 29, (2007)

18. Lu, C., Wu, H., Vemuri, S.: Neural network based on short term load forecasting, pp.336-342. IEEE Transactions on Power Systems, 8 (1), (1993) 